\title{
Sahayaka EOG based Voting System for Paralyzed People
}

\author{
Mr. Koushik Shridhar \\ Student \\ Electronics And Communication \\ SJCIT, Chikkaballapur \\ Karnataka, India \\ Mr. N Aravind \\ Student \\ Electronics And Communication \\ SJCIT, Chikkaballapur \\ Karnataka, India
}

\author{
Mr. Karan S Yadav \\ Student \\ Electronics And Communication \\ SJCIT, Chikkaballapur \\ Karnataka, India \\ Mr. P Umar Farook \\ Student \\ Electronics And Communication \\ SJCIT, Chikkaballapur \\ Karnataka, India
}

\author{
Dr. Bhaskar S \\ Professor \\ Electronics And Communication \\ SJCIT, Chikkaballapur \\ Karnataka, India
}

\begin{abstract}
Inspired by the recent advancement in sensor technology and learning about behaviour of human eye, in this paper we developed an system that would allow all physically challenged people to vote based on EOG signals. In this study, Electro-oculogram (EOG) based Human-Machine Interface (HMI) application is proposed for partial or completely paralyzed and physically limited people as Amyotrophic Lateral Sclerosis (ALS) patients. EOG-based systems are more efficient than electroencephalogram (EEG)-based systems as easy acquisition, higher amplitude, and also easily classified. An EOG is a method of tracking the ocular movement, based on the voltage changes that occur due to special orientation of the eye dipole. The resulting signal has a myriad of possible applications. In the designed system, Electro-oculogram signals consisted of vertical and horizontal eye movements are detected by using AgAgCl electrodes which placed around the eye. Then, by applying and filtering processes to the detected signals, Electro-oculogram signals at required amplitude levels are obtained at the output of analog stage. In consequence of digital processing of analog Electro-oculogram data with microcontroller unit, control signals for Human-Machine Interface applications are acquired. At signal processing stage, after preprocessing step for eye movements (vertical, horizontal and blink) maximum and minimum voltage amplitude values are detected. Using these values directly we can build the required e-voting circuit which would help paralyzed people to vote.
\end{abstract}

Keywords:- Paralyzed people, $\mathrm{Ag}-\mathrm{AgCl}$ electrode, Electro oculogram signal, filtering, Microcontroller, e-voting system

\section{INTRODUCTION}

Voting is a fundamental right of any citizen that enables them to choose the leaders of tomorrow. Voting is a basic process that keeps a nation's governmental system works. It enables the citizens to choose their own government. It also allows the people to choose their representatives in the government. Voting not only enables the citizens to vote for political parties, but it also helps them to realize the importance of citizenship. Each and every vote makes a huge change in growth of the nation.

According to the recent studies approximately 5.4 million people i.e. about $1.9 \%$ of the population all over the world are paralyzed and are not able to vote for their nation's growth. But even if they engage in voting, they are always required to be dependent on others to do the same. Therefore, in this project we help to find a suitable cost-effective solution for this problem in turn giving the paralyzed people a sense of independence in voting. To accomplish this we take the most active organ of majority of paralyzed people, i.e. eyes. By studying the vertical and horizontal eye movement and taking the EOG signals with help of cost efficient electrodes placed around the eye of a paralyzed person, and then processing this signal for voting [2].

It is a basic human need to operate few objects like household items which have made our everyday life easier. Normal people can easily use and operate electronic appliances like fan, light etc. But for a person with paralyses which hampers his movements, it is very difficult to use these appliances day-to-day [1]. So we use the EOG signals which helps the person to use and operate few basic electronic appliances.

\section{SYSTEM OVERVIEW}

\section{A. Ag/AgCl Electrodes:}

Surface $\mathrm{Ag} / \mathrm{AgCl}$ electrodes are the most common and favored electrodes in clinical measurements for recording biological signals such as ECG, EMG and EEG. One of the 
main advantages of using $\mathrm{Ag} / \mathrm{AgCl}$ electrodes is the low noise level it generates during biological signals recording. $\mathrm{Ag} / \mathrm{AgCl}$ electrodes generate lower electrode-skin interface impedance and lower electrode-skin interface impedance value than stainless steel electrodes. They are also considered as nonpolarizable electrodes; the non-polarizable nature of $\mathrm{Ag} / \mathrm{AgCl}$ electrodes allows the charges to cross the electrode-electrolyte interface unlike stainless steel electrodes. An silver-silver chloride ( $\mathrm{Ag} / \mathrm{AgCl}$ Electrodes) used for carrying EOG signal. EOG signal means potential between retina and cornea of an eye area for these electrodes are located near the eye area. .silver- silver chloride electrode provides accurate and clear transmission of surface biopotentials. We provide both DC and AC coupled Bio Amplifier for the acquisition of rapid eye movement along vertical and horizontal axes. These EOG signals are passed to the amplifier circuit. These electrodes are less expensive and comfortable to user [3].

\section{B. EOG Signal Acquisition \& amplification}

The EOG signal have potential ranges from $0.4 \mathrm{mV}$ to $1 \mathrm{mV}$ and a pair of electrodes are commonly used to detect this signal, but the voltage difference when there's an eye movement can be as small as just some micro volts. Due to the fact that an oscilloscope or a CPU cannot detect such small voltages, an EOG system must amplify those voltages in order to get a readable signal. Electrooculographic potential (EOG) presents a good face access, good accuracy and resolution, great range of eye displacements, works in real time and is cheap. In left gaze of the eye, the cornea approaches the electrode near the left eye, resulting in a positive going change and for right gaze, negative going change in the potential difference recorded from it [6].

An instrumentation amplifier is a type of differential amplifier that has been outfitted with input buffer, which eliminate the need for input impedance matching and thus make the amplifier particularly suitable for use in measurement and test equipment. A high pass filter is an electronic filter that passes high-frequency signals but attenuates signals with frequencies lower than the cutoff frequency. A low pass filter is a filter that passes low frequency signals with frequencies higher than the cutoff frequency. The actual amount of attenuation varies depending on specific filter design [2].

\section{Microcontroller ATmega8}

The ATmega8 provides the following features: 8 Kbytes of In-System Programmable Flash with Read-While Write capabilities, 512 bytes of EEPROM, 1 Kbyte of SRAM, 23 general purpose I/O lines, 32 general purpose working registers, three flexible Timer/Counters with compare modes, internal and external interrupts, a serial programmable USART, a byte oriented Two wire Serial Interface, a 6channel ADC (eight channels in TQFP and QFN/MLF packages) with 10-bit accuracy, a programmable Watchdog Timer with Internal Oscillator, an SPI serial port, and five software selectable power saving modes.

The ATmega8 is used in this project due to requirement of $\mathrm{ADC}$, the signal that we have taken from electrodes that are in analog form that's why we need to convert it in digital form. For that $\mathrm{ADC}$ is required.

\section{Arduino IDE}

Arduino IDE is a open source software that is mainly used for writing and compiling the code into Arduino module. It is ready to use immediately after installation. The main code, also known as a sketch, created on the IDE platform will ultimately generate a Hex File which is then transferred and uploaded in the controller on the board. The IDE environment mainly contains two basic parts: Editor and Compiler where former is used for writing the required code and later is used for compiling and uploading the code into the given Arduino Module. This environment supports both $\mathrm{C}$ and $\mathrm{C}++$ languages. Hence we are using this software to program the microcontroller.

\section{E. RF Transmission and Receiving}

This radio frequency (RF) transmission system employs Amplitude Shift Keying (ASK) with transmitter/receiver (Tx/Rx) pair operating at $434 \mathrm{MHz}$. The transmitter module takes serial input and transmits these signals through RF. The transmitted signals are received by the receiver module placed away from the source of transmission. This radio frequency (RF) transmission system employs Amplitude Shift Keying (ASK) with transmitter/receiver (Tx/Rx) pair operating at 434 MHz. The transmitter module takes serial input and transmits these signals through RF. The transmitted signals are received by the receiver module placed away from the source of transmission.

\section{SYSTEM ARCHITECTURE}

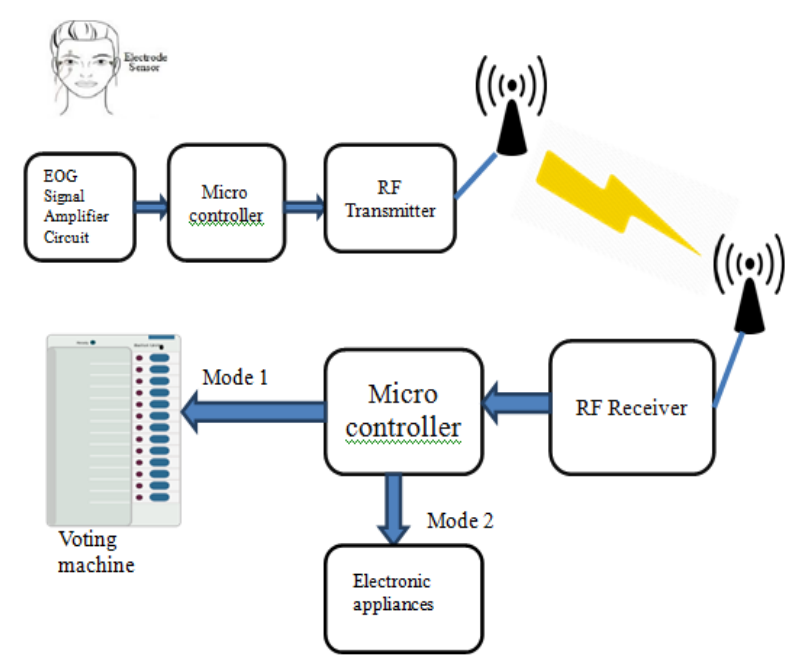

Fig.1: Block diagram of EOG Based Voting Machine. 
IV. FLOWCHART

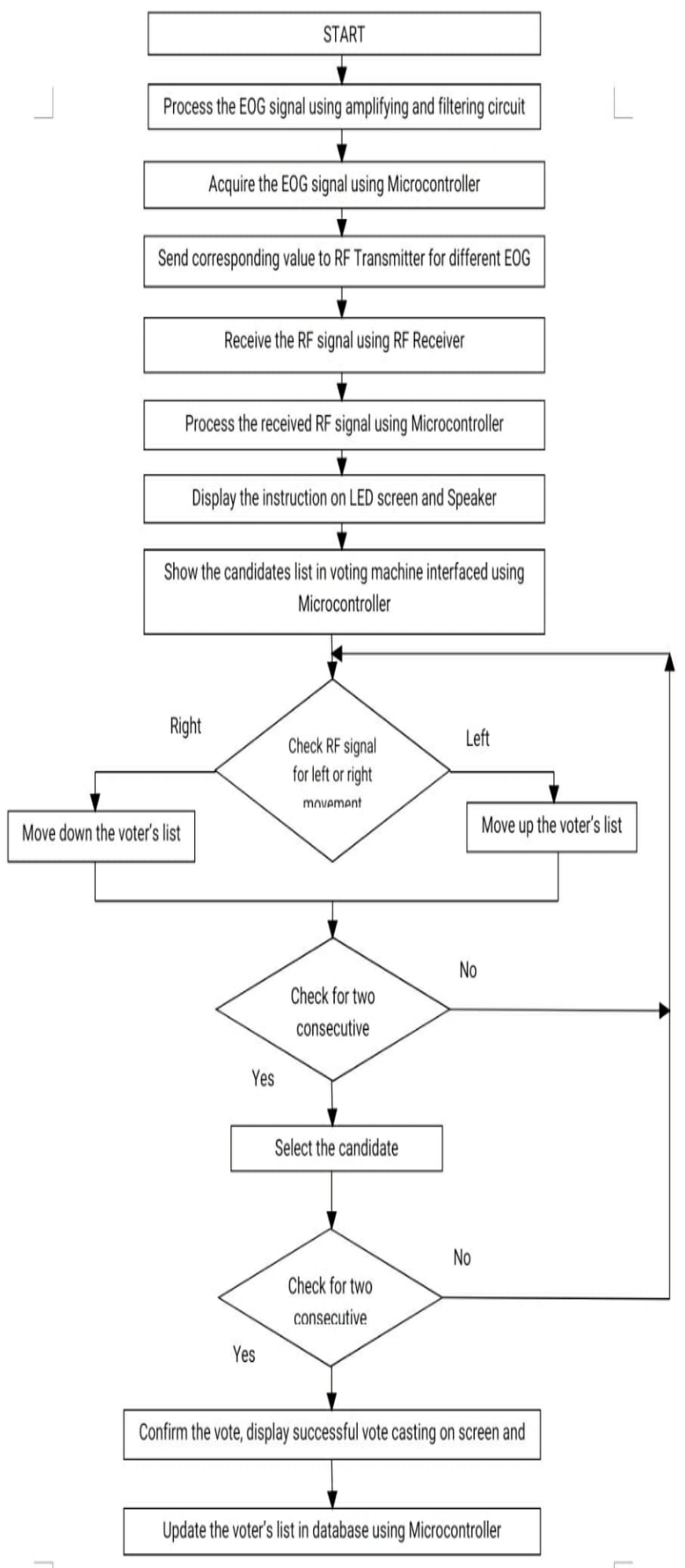

Fig.2: Flowchart representation

\section{ADVANTAGE}

- EOG is easy to use in patients confined to bed (paralyzed and physically limited people).

- The EOG is linearly proportional to eye displacement.

- The signals are measured with respect to head.

- The EOG is most inexpensive eye movement recording system.

\section{CONCLUSION}

In this project, we try to assist the physically-challenged people to vote using their eyes. One of the major aim of our work is to build a system that gives a reasonably fast output and which is very economical to be commercially manufactured and used for the purpose.

In conclusion, this method of acquiring EOG signal and using it for various fields of application seems to reduce the design complexity of the system and improve the signal efficiency to the required level. There are many other alternate methods and circuits available for acquiring the EOG signal in more a accurate manner, but this project aims at minimizing the cost to great extent. Therefore, there has to some compromise in signal efficiency and system response.

The impact of the basic circuits used for signal acquisition makes it a bigger contributor to cut the cost of the project as a whole. Since the components used in this project are mostly separately available in the market, it makes it even easier to repair and restore the system in case of any failure.

\section{ACKNOWLEDGEMENT}

We express our sincere thanks to our Department and our Institute for fostering an excellent academic environment which made this endeavor fruitful.

We express wholehearted gratitude to Dr. Bhaskar S. We wish to acknowledge his help in making our task easy by giving us his valuable advice and encouragement.

We would be very pleased to express our heart full thanks to the teaching and non-teaching staff of the department of Electronics and Communication Engineering, S J C Institute of Technology for their motivation and support.

\section{REFERENCES}

[1] Nikesh Borkar, Tridev Dongare, Pratik Chahande, Jay Bonsod "Microcontroller Based EOG and Accelometer Guide Wheelchair", IRJET, volume 2, issue 6 1432-1436 Mar-2018

[2] Md Fahim, Md Ahsan, Nazmus Sakib -"Design and Development of an EOG-based System to Control Electric Wheelchair for Paralysed People”, doi :ICREST.86442495, Jan 2019.

[3] Rui Zhang, Kai Li, Xichun Zhang -“An EOG Based Human-Machine Interface to Control a Smart-Home Environment for Patients with Severe Spinal Cord Injuries", ASMA transaction 16:870-873, IEEE 2019.

[4] Min-Ho Lee, John Williamson, Siamac Fazli -“A High Performance Spelling System based on EEG-EOG Signals with Visual Feedback", IEEE Transaction volume 10 issue 2 ISSN-6491175, ,IEEE-2018.

[5] Anson Bastes, Siddarth Alhat, M.S Panse -"Speech Assistive Communication System using EOG", ICICCS-2018.

[6] Koushik Sarathy Sridharan,- Real-time acquisition and analysis of Electro-oculography signals, Masters thesis, 2012. 